\title{
PROPOSTA DE UM INSTRUMENTO DE COLETA DE DADOS EVIDENCIANDO A RELAÇÃO DA DIABETES MELLITUS TIPO 1 E DOENÇA TIREOIDIANA AUTOIMUNE
}

\author{
Alaíde Policarpo Costa ${ }^{a}$ \\ Márcio Osvan Rezende Rocha Xaviera \\ Isabella Lorrayne Silva ${ }^{a}$ \\ Marta Lamounier Moura Vargas Corgozinhoa \\ Lindiane Lopes Fereira ${ }^{a}$ \\ Rafaela de Oliveira Silva ${ }^{a}$ \\ Sabrynna Brito Oliveiraa,b,
}

\begin{abstract}
a Núcleo de Biociências, Centro Universitário Metodista Izabela Hendrix, Rua da Bahia, 2020, Bairro Lourdes, Belo Horizonte - MG, 30160-012

b Departamento de Microbiologia, Instituto de Ciências Biológicas, Universidade Federal de Minas Gerais, Av. Pres. Antônio Carlos, 6627 - Pampulha, Belo Horizonte - MG, 31270-901.
\end{abstract}

\section{RESUMO}

Introdução: O Diabetes Mellitus tipo 1 (DM1) é doença caracterizada pela insuficiência de insulina devido à perda das células $\beta$, levando à hiperglicemia. $A$ incidência do DM1 tem sido frequentemente associada a doenças autoimunes, como as tireoidites. No entanto, tem-se observado uma maior propensão de pacientes com disfunções tireoidianas em desenvolver Doenças Tireoidianas Autoimunes (DAT) e a inexistência de consenso, na literatura, sobre os métodos de identificação da disfunção tireoidiana em pacientes com DM1. Objetivo: Objetivou-se criar instrumento de coleta de dados para avaliar a incidência, prevalência e identificar o perfil clínico de pacientes com DM1 que desenvolveram alterações na tireoide, de modo a observar a sua reprodutibilidade na obtenção de dados epidemiológicos. Metodologia: Trata-se de um estudo do tipo metodológico, estruturado em quatro etapas: criação de um instrumento de coleta de dados, validação do conteúdo, refinamento do instrumento e simulação de coleta de dados. Resultados: O primeiro instrumento de coleta de dados elaborado com base na literatura científica retornou aos pesquisadores com a recomendação de inserção das seguintes variáveis: Anti-TPO, Anti-GAD, tabagismo, etilismo, outras doenças autoimunes, gestações. Ao utilizar esse instrumento em uma simulação, percebeu-se que poderiam ser incluídos os tópicos: complicação, impacto da doença, limitações, condição socioeconômica, para se ter uma visão mais ampla do perfil do paciente e da prevalência das doenças autoimunes. Conclusão: $O$ estudo demonstrou ser fundamental o aprimoramento das fichas de coleta de dados a fim de tornar mais preciso o rastreamento de DTA logo após o diagnóstico de DM1.

Palavras-chave: Diabetes Mellitus Tipo 1. Doenças autoimunes. Tireoide. Prevalência.

*Autor correspondente: Sabrynna Brito Oliveira, Doutora em Microbiologia. Enderço: Av. Pres. Antônio Carlos, 6627 - Pampulha, Belo Horizonte - MG, 31270-901, ICB - bloco C4 sala 109. Telefone de contato: (31) 992822768; E-mail de contato: sabrynnabrito@gmail.com. 


\section{INTRODUÇÃO}

O diabetes mellitus tipo 1 (DM1), conhecido como diabetes autoimune, é uma doença caracterizada pela insuficiência de insulina devido à perda das células $\beta$, células endócrinas nas ilhotas de Langerhans do pâncreas responsáveis pela síntese e secreção do hormônio insulina, levando à hiperglicemia. Sua etiologia está relacionada a fatores genéticos derivados da herança poligênica e, em grande parte dos casos, podem estar envolvidos fatores ambientais e aspectos nutricionais (KHAN, 2019).

O DM1 é frequentemente associado a outros distúrbios autoimunes, tais como tireoidite de Hashimoto, doença de Graves, doença de Addison, vitiligo, insuficiência ovariana primária, anemia perniciosa e doença celíaca. Por essa razão, a American Diabetes Association's (ADA's, 2017) recomenda o rastreio de doenças autoimunes da tireoide e doenças celíacas logo após o diagnóstico (ASD's, 2017).

Os distúrbios da tireoide acometem cerca de $12 \%$ da população em geral e sua prevalência é maior em idosos do sexo feminino e nos indivíduos com anticorpos antitireoidianos (AAT). Além disso, a associação entre DM1 e disfunções tireoidianas pode interferir nos níveis de Triiodotironina (T3) e Tiroxina (T4) livres, bem como no Hormônio Estimulador da Tireoide (TSH), fazendo que prevaleçam doenças tireoidianas em pacientes com DM1 (NEDERSTIGT, 2016)

Ademais, pacientes com DM1 têm maior risco de desenvolver doenças tireoidianas autoimune (DTA), podendo ser explicada a partir da relação dos genes compartilhados tanto para o DM1 como para as tireopatias, onde cerca de $12 \%$ a $24 \%$ das mulheres e $6 \%$ dos homens com DM1 desenvolvem hipotireoidismo. Em contrapartida, a prevalência do hipertireoidismo é menor que $1,7 \%$, alguns estudos demonstram que cerca de $50 \%$ dos pacientes que possuem anticorpos antiperoxidase (APTO) desenvolveram alguma alteração na tireoide em um período de 10 anos do diagnóstico do DM1 (SMITHSON, 1998; EISENBARTH et al., 2004; SILVA, 2005).

Assim, a triagem de tireopatias em pacientes com DM1 é indicada para prevenir o aumento da disfunção tireoidiana clínica. Sendo assim, o diagnóstico e o tratamento do hipotireoidismo anteparam o aparecimento de dislipidemia, impedindo qualquer disfunção endotelial e agravamento das complicações macroangiopáticas, e evitam os efeitos adversos da diminuição dos hormônios tireoidianos sobre o controle glicêmico (maior tendência à hipoglicemia) e o crescimento em crianças (SILVA, 2005).

O diagnóstico e tratamento do hipertireoidismo impedem o agravamento da intolerância à glicose, o desenvolvimento de osteoporose e fibrilação atrial. Como os pacientes com DM1 desenvolvem disfunção tireoidiana em idade mais precoce que a população geral, recomenda-se que todos sejam investigados independentemente da idade. Não existe consenso na literatura sobre os métodos de identificação da disfunção tireoidiana em pacientes com DM1 (GHARIB, 2005). A dosagem dos anticorpos antitireoidianos como única ferramenta diagnóstica pode não detectar $28 \%$ dos pacientes com hipotireoidismo subclínico (SOUZA, 2004).

Dessa forma, a fim de conhecer qual é a incidência de DTA em pacientes com DM1 e seu impacto no perfil de morbidade da população, este estudo visou a elaboração de um instrumento de coleta de dados voltado para a investigação da incidência e prevalência de DTA em pacientes com DM1.

\section{METODOLOGIA}

Trata-se de um estudo do tipo metodológico, desenvolvido de janeiro à dezembro de 2021, que foi estruturado em quatro etapas: criação de um instrumento de coleta de dados, validação do conteúdo, refinamento do instrumento e simulação de coleta de dados.

\subsection{PRIMEIRA ETAPA: CRIAÇÃO DE UM INSTRUMENTO DE COLETA DE DADOS}

Essa etapa compreendeu um levantamento bibliográfico em periódicos indexados nas bases de dados da LILACS (Literatura Latino-Americana e do Caribe em 
Ciências da Saúde) e MEDLINE (Literatura Internacional em Ciências da Saúde e Biomédica), utilizando como descritores: "Diabetes Mellitus Tipo 1", "Doenças autoimunes", "Tireoide", "Prevalência". Foram consideradas pesquisas publicadas em português, nos últimos 10 anos, disponibilizadas na íntegra nas plataformas acima mencionadas. Além disso, foram considerados livros da área de endocrinologia, metabologia e bioquímica para subsidiar a construção do instrumento de coleta de dados. $O$ instrumento inicial foi organizado em três sessões: 1) Dados sociais e demográficos; 2) Dados clínicos; 3) Dados Laboratoriais.

\subsection{SEGUNDA ETAPA: VALIDAÇÃO DE CONTEÚDO}

$\mathrm{Na}$ segunda fase, o instrumento elaborado foi submetido aos especialistas para avaliação do conteúdo contemplado e a forma de apresentação dessa ferramenta. Considerou-se especialista o médico com residência ou especialização na área de endocrinologia/metabologia, com experiência profissional de pelo menos dois anos de atendimento ao público.

Os especialistas foram identificados por meio de busca ativa nos consultórios particulares e públicos da cidade de Belo Horizonte - MG, além de busca nas plataformas Lattes e de divulgação médica. Os médicos foram convidados por meio de carta enviada por correio comum ou eletrônico. $\mathrm{Na}$ carta eram explicados o título e os objetivos do estudo e justificativa do processo de validação. Foram convidados 30 médicos, dos quais 2 responderam ao convite e aceitaram participar do processo. Enviou-se posteriormente o termo de consentimento livre e esclarecido, roteiro sobre o processo de validação e instrumento de avaliação. Estabeleceu-se um prazo de 30 dias para devolução do material por meio de correio comum ou eletrônico de acordo com a preferência de cada participante. Os especialistas opinaram sobre a adequação de cada item que compunha a seção e do conjunto da seção com base nos critérios de simplicidade, amplitude, clareza e precisão (8). Foi solicitada a anuência em participar por meio da assinatura do termo de consentimento livre e esclarecido. Foi garantindo o sigilo da identidade e assegurado à liberdade para que qualquer integrante deixasse 0 grupo a qualquer momento.

\subsection{TERCEIRA ETAPA: REFINAMENTO DO INSTRUMENTO}

Após a avaliação pelos especialistas, o documento foi devolvido aos autores contendo as adequações recomendadas. Houve uma reformulação da ficha-roteiro com as alterações sugeridas pelas médicas.

\subsection{QUARTA ETAPA: SIMULAÇÃO DE COLETA DE DADOS}

Após a adequação do instrumento de coleta de dados, optou-se por simular uma coleta de dados a fim de testar o instrumento e comprovar a sua validação. Nessa etapa foram utilizados prontuários de pacientes diagnosticados com DM1 e/ou DTA atendidos por uma das médicas que fez parte da etapa 2 . Foram incluídos neste estudo os prontuários $\mathrm{e}$ fichas de acompanhamento de pacientes com 18 anos ou mais, independentemente do sexo, residentes em Belo Horizonte e que estejam em acompanhamento há pelo menos 5 anos com o diagnóstico de DM1 e/ou DTA. Não foram contabilizados os casos oriundos de pacientes com menos de 18 anos, residentes em outros municípios, acompanhados há menos de 5 anos, mesmo com o diagnóstico de interesse e cuja causa principal do acompanhamento não for DM1 e/ou DTA e nem os prontuários incompletos. Foram coletados os dados clínicos, laboratoriais e sociais referentes ao acompanhamento de casos de DM1 com posterior descoberta de DTA ou de pacientes com DTA que tivessem DM1 subclínica e confirmada durante o tratamento da DTA, a conduta abordada e desfecho do caso.

Os dados acessados pelos pesquisadores foram compilados de dados fornecidos pelas médicas, o que impossibilitou o acesso direto aos pacientes ou aos seus dados pessoais completos. Dessa forma, com base na Resolução CNS 466/12 (item IV.8), justificou-se a dispensa do Termo de Consentimento Livre e Esclarecido - TCLE dos 
pacientes. O projeto foi aprovado pelo Comitê de Ética em Pesquisa do Centro Universitário Metodista Izabela Hendrix (CAAE 45384621.0.0000.5096).

\section{RESULTADOS E DISCUSSÃO}

O primeiro instrumento de coleta de dados foi criado pelos autores com base em artigos que desenvolveram trabalhos de levantamento de dados envolvendo DM1 e DTA. A primeira versão contou com questões discursivas organizadas em três sessões: 1) Dados sociais e demográficos; 2) Dados clínicos; 3) Dados laboratoriais.

A segunda versão incorporou com várias sugestões de melhoria vindas dos especialistas, tanto no conteúdo como na aparência. Nenhuma variável da ficha 1 foi descartada. As variáveis incorporadas ao instrumento estão sinalizadas em negrito. A comparação entre os instrumentos pode ser mais bem visualizada na Tabela 1.

Tabela 1. Evolução da construção de um instrumento de coleta de dados criado para investigação da relação entre Diabetes Mellitus tipo 1 e doenças tireoidianas autoimunes.

\begin{tabular}{|c|c|c|}
\hline & ICD 1 & ICD 2 \\
\hline SESSÃO & \multicolumn{2}{|c|}{ VARIÁVEIS } \\
\hline $\begin{array}{l}1 \text { - Dados } \\
\text { sociais e de- } \\
\text { mográficos }\end{array}$ & $\begin{array}{l}\text { Idade; Sexo; Escolaridade; } \\
\text { Município de residência. }\end{array}$ & $\begin{array}{l}\text { Idem ao ICD } 1 \\
\text { Adição da "Naturalidade" }\end{array}$ \\
\hline $\begin{array}{l}2 \text { - Dados clí- } \\
\text { nicos }\end{array}$ & $\begin{array}{l}\text { Histórico familiar } \\
\text { Fatores de risco } \\
\text { Comorbidades } \\
\text { Data/período do diagnóstico } \\
\text { DTA } \\
\text { Portador de DM1 no momen- } \\
\text { to do diagnóstico de DTA; } \\
\text { Desfecho clínico do caso. }\end{array}$ & $\begin{array}{l}\text { Transformação de três em múltipla } \\
\text { escolha: } \\
\text { Histórico familiar: } \\
\text { - } \quad \text { Possui algum parente de pri- } \\
\text { meiro grau com DM1? } \\
\text { - Possui algum parente de pri- } \\
\text { - } \quad \text { Posiro grau com DTA? } \\
\text { meiro grau com alguma alte- } \\
\text { ração autoimune? } \\
\text { Fatores de risco: } \\
\text { - } \quad \text { etilismo } \\
\text { - } \text { tabagismo } \\
\text { - dedentarismo } \\
\text { - } \text { deficiência de vitamina D } \\
\text { Coutras doenças autoimunes } \\
\text { Comorbidades }\end{array}$ \\
\hline $\begin{array}{l}3 \text { - Dados la- } \\
\text { boratoriais }\end{array}$ & $\begin{array}{l}\text { Resultados de exames im- } \\
\text { portantes para a compreen- } \\
\text { são/acompanhamento do } \\
\text { caso. }\end{array}$ & $\begin{array}{l}\text { Investigação dos principais exames } \\
\text { associados: } \\
\text { - } \quad \text { Anti-TPO; Anti-GAD; } \\
\text { - } \text { IAA; IA2 } \\
\text { - H4 Livre; T3; TSH; } \\
\text { HbA1c; TTOG; Glicemia em } \\
\text { - } \text { Outros: resultados de exa- } \\
\text { mes importantes para a com- } \\
\text { preensão/acompanhamento } \\
\text { do caso. }\end{array}$ \\
\hline
\end{tabular}

*ICD: Instrumento de coleta de dados. ICD1 - criado pelos autores. ICD2 - revisado pelos especialistas. Fonte: os autores 
A sessão 1 abordou tópicos como idade, sexo, escolaridade e local de residência. A inclusão do fator "idade" e o fator "sexo" do paciente fez-se necessário para saber se há influência na determinação de alguma síndrome subsequente ao DM1. Em estudos realizados por Smithson (1998) e Eisenbarth e colaboradores (2004) constatou-se que a maior prevalência está entre o sexo feminino (razão homem $/$ mulher $=1 / 3$ ) e na fase adulta, entre 20 e 60 anos de idade.

A sessão 2 abordou os temas histórico familiar, fatores de risco, comorbidades e aspectos do diagnóstico inicial. Justifica-se a discussão do tópico "histórico familiar" por se tratar de doenças com predisposição genética. O histórico familiar torna-se importante para a detecção e prevenção de doenças autoimunes, pois é um registro que mapeia as informações clínicas e biológicas do paciente e seus parentes, principalmente de primeiro grau. Além disso, informa quanto aos hábitos e costumes herdados dos familiares que podem ser fatores predisponentes ao desenvolvimento de doenças, a exemplo do tabagismo e etilismo.

Sobre hereditariedade genética, Decochez e colaboradores (2002) relataram em seu estudo que pacientes que possuem algum parente com DM1 e expressam altos níveis de IAA, GAD e IA2A tendem a ter $75 \%$ de risco de desenvolverem DM1 num período de 5 anos, já em comparação aos que expressam somente um dos anticorpos, o risco é de 10 a $25 \%$.

Eisenbarth e colaboradores (2004) relataram que muitas doenças autoimunes podem ser causadas por mutações de gene supressor autoimune, no qual pacientes afetados têm a predisposição de "desenvolver várias doenças autoimunes adicionais ao longo do tempo" como DM1, vitiligo, hipotireoidismo, hepatite, anemia perniciosa entre outras. Fato ratificado pelo presente estudo, pois todos os prontuários apresentaram familiares com DM1, tireoidite de Hashimoto, doença de Graves (DG), Vitiligo e Artrite Reumatoide.

No tópico "fatores de risco", buscou-se investigar quais são os fatores que aumentam o risco de pacientes com DM1 e/ou tireoidites desenvolverem outros tipos de doenças ou alterações clínicas.

A sessão 3 foi voltada aos exames laboratoriais. Para a avaliação de possíveis alterações associadas às patologias estudadas, faz-se importante a dosagem de anticorpos específicos, vale dizer, o anti-TPO (para tireoidite de Hashimoto), TSH (para doença de Graves) em pacientes que possuem DM1 e podem desenvolver alguma alteração autoimune na tireoide. Por outro lado, estudos sugerem que o anti-insulina (IAA), antiácido glutâmico descarboxilase-65 (GAD) e antitirosina fosfatase IA-2 (IA2A) são fatores de risco potencialmente elevados para 0 desenvolvimento do DM1 no período de 5 anos (DECOCHEZ et al., 2002). Nos dados relativos aos exames laboratoriais presentes nas fichas, constatou-se recorrência na solicitação de cálculos/dosagem de anticorpos anti-TPO e anti-GAD, os quais, por conseguinte, atestam ser relevantes para o diagnóstico.

As alterações que ocorrem na tireoide iniciam-se com uma pequena infiltração da glândula por linfócitos e macrófagos, que podem gerar atrofia na glândula tireoide e destruição do tecido, levando à clássica deficiência hormonal. Em pacientes que possuem DM1, o cálculo dos anticorpos Antiperoxidase (Anti-TPO) para a tireoide e Anti-ácido glutâmico (Anti-GAD) para o pâncreas aparecem como um manejo trivial para os diagnósticos (DRIESSCHE et al.,2009).

Segundo Giovinazzo et al., (2017), o "cálculo de vitamina D" se faz importante devido à sua participação no sistema imune inato e adaptativo, e sua ausência ou insuficiência está associada a um alto risco no desenvolvimento de doenças autoimunes.

A maioria dos estudos envolvendo o uso da vitamina $\mathrm{D}$ se associa ao DM1, devido ao impacto na sensibilidade à insulina. Existem evidências clínicas que sugerem que os níveis de vit. $D$ estão diretamente ligados às alterações tanto nos pacientes com DM1 quanto naqueles com DTA, por meio da sua atuação nas células $T$ helper-2 e células $\beta$ (KIVITY et al., 2011).

Após a reformulação da ficha, o 
instrumento foi utilizado em uma simulação de coleta de dados utilizando seis prontuários de pacientes atendidos por uma das médicas, que nos cedeu gentilmente. É importante ressaltar que por ser um estudo de validação, com base em prontuários pré-selecionados, algumas das variáveis da seção "Dados sociais e demográficos" não foram coletadas.

Os prontuários referem-se a pacientes do sexo feminino, das quais três estavam na faixa etária de 18 a 28 anos, duas na faixa etária de 40 a 50 anos e uma entre 72 e 82 . Dos seis prontuários disponibilizados, quatro foram de pacientes com diagnóstico inicial de DM1 e dois de pacientes diagnosticadas inicialmente com DTA.

Sobre o diagnóstico de DM1, verificou-se que quatro pacientes receberam diagnóstico inicial de DM1 na faixa etária de 18 a 28 anos e duas de 29 a 39. Nos prontuários referentes aos diagnósticos da DTA, observou-se que as duas pacientes foram diagnosticadas inicialmente com DTA aos 15 anos. Todas as pacientes têm nível superior de escolaridade

As pacientes apresentaram histórico familiar de doença autoimune, das quais três possuem vários casos de DM1 e Hashimoto na família, duas possuem pai portadores da Doença de Graves (DG) e Vitiligo, e uma possui mãe diagnosticada com Artrite Reumatoide e filha com DM1.

Em relação aos fatores de risco, todas pacientes apresentaram a presença de hipercolesterolemia familiar. Quanto à avaliação dos dados sociais, nenhuma paciente apontou tabagismo, mas todas notificaram etilismo social.

No que concerne a comorbidades, uma paciente apresentou infecções urinárias de repetição, duas apresentaram neuropatia diabética e retinopatia diabética, uma tem hipertensão arterial, uma tem nefropatia diabética, uma apresentou edema macular diabético e duas não apresentaram ocorrências. A respeito de outras doenças autoimunes, verificou-se o registro de uma paciente com vitiligo, uma com menopausa precoce, uma com artrite reumatoide e três não apresentaram ocorrências. No tocante a gestações, quatro pacientes não tiveram gravidez e duas informaram 2 gestações cada uma. Não se fez possível verificar a relação entre gravidez e alterações autoimunes nos prontuários disponibilizados para estudo.

No momento do diagnóstico de DTA, somente quatro pacientes apresentaram ser portadoras de DM1. O desenvolvimento da DTA nas pacientes com DM1 apresentou intervalo variável de 5 a 23 anos.

Sobre os exames laboratoriais em análise nos prontuários, observou-se que na dosagem de anticorpos antitireoperoxidase (anti-TPO) cinco pacientes apresentaram resultados positivos. Desses cinco casos, três pacientes notificaram DM1 e Tireoidite de Hashimoto no Histórico Familiar, e duas pacientes apontaram doença de Graves e vitiligo. Uma paciente teve resultado negativo para o anti-TPO, consta no seu Histórico Familiar, DM1 e Hashimoto.

$\mathrm{Na}$ dosagem de Anti-ácido glutâmico (Anti-GAD), cinco pacientes apresentaram resultados positivos, desses casos, três apontaram DM1 e Hashimoto no Histórico Familiar e dois apresentaram doença de Graves e vitiligo, e o resultado de uma paciente não foi disponibilizado. Nos exames de imagem, cinco pacientes apresentaram ultrassonografia compatível com Tireoidite de Hashimoto.

Em 2009, Driessche e colaboradores demonstraram em seu artigo que até um terço dos pacientes com DM1 desenvolvem uma síndrome poliglandular autoimune, que pode ser tireoidite de Hashimoto ou Graves, gastrite autoimune, vitiligo, anemia perniciosa, doença celíaca, dentre outras. A saber, por exemplo, que o "hipotireoidismo de Hashimoto pode causar ganho de peso, hiperlipidemia, bócio e pode afetar o controle do diabetes, a menstruação e o resultado da gravidez".

Os fatos acima foram constatados nas fichas de coleta de dados, onde houve relatos de hipercolesterolemia familiar, vitiligo, menopausa precoce e complicações na gravidez. O que levou à dedução, também, que o DM1 e as tireoidites autoimunes podem ter provocado as comorbidades e/ou complicações elencadas nas fichas de dados como hipertensão arterial, neuropatia periférica, infecções urinárias de repetição, 
retinopatia diabética, nefropatia diabética, edema macular diabético e artrite reumatoide. Os dados relacionados acima podem ser

Tabela 2. Dados coletados dos prontuários melhor visualizados na Tabela 2.

\begin{tabular}{|c|c|c|c|c|c|c|c|c|c|}
\hline & $\begin{array}{l}\text { Faixa } \\
\text { etária }\end{array}$ & $\begin{array}{l}\text { Diagnóstico } \\
\text { inicial }\end{array}$ & $\begin{array}{l}\text { Idade do } \\
\text { diagnóstico } \\
\text { do DM1 }\end{array}$ & $\begin{array}{l}\text { Histórico } \\
\text { familiar de } \\
\text { doenças } \\
\text { autoimunes }\end{array}$ & $\begin{array}{l}\text { Fator } \\
\text { de } \\
\text { Risco }\end{array}$ & $\begin{array}{l}\text { Comorbi- } \\
\text { dade }\end{array}$ & $\begin{array}{l}\text { Outras } \\
\text { doenças } \\
\text { autoimunes }\end{array}$ & $\begin{array}{l}\text { Anti - } \\
\text { TPO }\end{array}$ & $\begin{array}{l}\text { Anti - } \\
\text { GAD }\end{array}$ \\
\hline P 1 & $18-28$ & DM1 & $18-28$ & DM1 / TH & $\mathrm{HF} / \mathrm{ES}$ & ND/RD/EM & - & POS & POS \\
\hline P 2 & $18-28$ & DM1 & $18-28$ & DG / VIT & $\mathrm{HF} / \mathrm{ES}$ & - & - & POS & POS \\
\hline P 3 & $18-28$ & DM1 & $29-39$ & DG / VIT & $\mathrm{HF} / \mathrm{ES}$ & - & - & POS & POS \\
\hline P 4 & $40-50$ & DM1 & $29-39$ & DM1 / TH & $\mathrm{HF} / \mathrm{ES}$ & RD/NFD & AR & POS & NEG \\
\hline P 5 & $40-50$ & DTA & 15 & AR / DM1 & HF/ES & IUR & $\begin{array}{l}\text { Menopausa } \\
\text { precoce }\end{array}$ & NEG & POS \\
\hline P 6 & $72-82$ & DTA & 15 & $\mathrm{DM} 1 / \mathrm{TH}$ & $\mathrm{HF} / \mathrm{ES}$ & HA/NP & VIT & POS & POS \\
\hline
\end{tabular}

DM1 - Diabetes Mellitus tipo 1/ DTA - Doença Tireoidianas Autoimunes/ TH - Tireoidite de Hashimoto/ DG - Doença de Graves/ VIT - Vitiligo/ AR - Artrite Reumatoide/ ES - Etilismo Social/ HF - Hipercolesterolemia familiar/ IUR Infecção urinaria de repetição/ ND - Neuropatia Diabética/ RD - Retinopatia Diabética/ NFD - Nefropatia Diabética/ HA - Hipertensão Arterial/ NP - Neuropatia periférica/ EM - Edema macular/ POS - Positivo/ NEG - Negativo. Fonte: os autores.

Após as três primeiras etapas do estudo, o instrumento de coleta de dados foi utilizado em uma simulação com base em prontuários de seis pacientes do sexo feminino, com suas autorizações. Conquanto os dados obtidos nas fichas não tenham possibilitado comparação de percentual entre os sexos por causa no número amostral pequeno, a literatura também registra o acometimento do sexo masculino ainda que em menor porcentagem. $\mathrm{O}$ mesmo se estende à análise da faixa etária, que revelou que o diagnóstico de DM1 e DTA aparenta ser mais presente no público entre 18 anos e 59 anos, em consonância com a literatura citada, que aponta para idades entre 20 anos e 60 anos.

No que se refere ao Brasil, onde existem muitas disparidades educacionais, o grau de escolaridade não pode ser desconsiderado em eventual estudo, tendo em vista que, em tese, pessoas com maior nível de instrução, com maior acesso à educação de qualidade e aos meios preventivos de saúde, estão mais

conscientizados a respeito dos riscos de doenças em geral e genéticas em particular. A teor das fichas, verificou-se que as pacientes tinham graduação em nível superior, o que obstou a análise de resultados entre pacientes de diferentes níveis de escolaridade. Tendo em vista que as médicas parceiras têm por pacientes pessoas com razoável padrão de vida, supõe-se que o nível de escolaridade também esteja, em alguma medida, associado à condição socioeconômica.

O estudo evidenciou a necessidade de se incluir, na ficha-roteiro, tópico relacionado à "condição socioeconômica" dos pacientes, a fim de se verificar se, de fato, as condições socioeconômicas caminham paralelamente à escolaridade e, em que medida, esses fatores, conjunta ou isoladamente, podem influenciar nos níveis de profilaxia e cuidados pessoais.

Com efeito, tendo em vista a elaboração de política-pública voltada ao DM1, há que se perquirir, além do grau de instrução, qual o nível de acesso aos meios preventivos e 
curativos à saúde, a fim de que o Estado possa alocar recursos (financeiros, humanos) para locais onde se identifique maior vulnerabilidade.

Assim, a análise dos resultados obtidos na etapa quatro, os autores perceberam que outras variáveis deveriam ser incluídas para a versão final (apêndice 1) da ficha: complicações, impactos da doença e condição socioeconômica.

\section{CONSIDERAÇÕES FINAIS}

Este estudo demonstrou ser mister o aprimoramento de instrumento de coleta de dados a fim de tornar mais preciso o rastreamento de DTA logo após o diagnóstico de DM1 e vice-versa. Outrossim, o estudo evidenciou a relevância da inclusão de especialistas, tanto para o aprimoramento das fichas quanto para a interpretação dos dados coletados com vista ao diagnóstico de alterações tireoidianas relacionadas ao DM1. Ao final, foi demonstrado que a elaboração de fichas mais completas pode auxiliar a elucidação e o acompanhamento de casos, a melhoria e o direcionamento das ações de controle dessas doenças, assim como contribuir para a qualificação acadêmica e profissional dos participantes do presente trabalho.

Um estudo prospectivo há der ser realizado em um hospital referência, de grande porte, em Minas Gerais, a fim de demonstrar se o modelo de instrumento de coleta de dados aqui desenvolvido ensejará resultados semelhantes aos da literatura no quesito incidência e prevalência de DM1 e DTA, ou se, uma vez aplicado em larga escala e nas dependências de estabelecimento da rede pública de saúde, far-se-á necessário o acréscimo de outros dados para conformar o modelo ao perfil do público assistido.

\section{ONFLITO DE INTERESSE}

Os autores afirmam que não há conflito de interesse na pesquisa.

\section{REFERÊNCIAS}

AMERICAN DIABETES ASSOCIATION (United States). American Diabetes Association. Standards of Medical Care in Diabetes2017 Abridged for Primary CareProviders. 2017. Disponível em: https:// clinical.diabetesjournals.org/content/35/1/5. Acesso em: 26 set. 2020.

DECOCHEZ K, de Leeuw IH, Keymeulen $B$, et al. IA-2 autoantibodies predict impending type I diabetes in siblings of patients. Diabetologia. 2002; 45:1658-66. Disponível em: https:// pubmed.ncbi.nlm.nih.gov/ 12488955/. Acesso em: 06 set 2021

EISENBARTH GS, Gottlieb PA. Autoimmune Polyendocrine Syndromes. N Engl J Med. 2004; 350:2068-79. Disponível em: https://pubmed.ncbi.nlm.nih.gov/ 15141045/. Acesso em: 08 set 2021

GHARIB, Hossein et al. CONSENSUS STATEMENT: Subclinical Thyroid Dysfunction: A Joint Statement on Management from the American Association of Clinical Endocrinologists, the American Thyroid Association, and The Endocrine Society. 2005. Disponível em: https:// pubmed.ncbi.nlm.nih.gov/15687817/. Acesso em: 27 set. 2020 .

GIOVINAZZO S, Vicchio TM, Certo R, et al. Vitamin $D$ receptor gene polymorphisms/ haplotypes and serum 25(OH)D3 levels in Hashimoto's thyroiditis. Endocrine 2017; 55: 599-606. Disponível em: https:// pubmed.ncbi.nlm.nih.gov/27043843/. Acesso em: 08 set 2021.

KHAN, Gohar et al. Thyroid dysfunction in patients with type 1 Diabetes. 2019. Disponível em: https://jpmi.org.pk/index.php/ jpmi/article/view/2315. Acesso em: 27 set. 2020. 
KIVITY S, AGMON-LEVIN, N; ZISAPPL, M. et al. Vitamin $D$ and autoimmune thyroid diseases.

Cell Mol Immunol. 2011; 8: 243-247. Disponível em: https:// pubmed.ncbi.nlm.nih.gov/21278761/. Acesso em: 18 set 2021.

NEDERSTIGT, $C$. et al. Incidence and prevalence of thyroid dysfunction in type 1 diabetes. 2016. Disponível em: https:// pubmed.ncbi.nlm.nih.gov/26868720/. Acesso em: 28 set. 2020 .

SILVA, Regina do Carmo. Importância da Avaliação da Função Tireoidiana em Pacientes Com Diabetes Mellitus. 2005. Disponível em: https://doi.org/10.1590/ S0004-27302005000200003. Acesso em: 27 set. 2020.

SMITHSON, M.J. Screening for thyroid dysfunction in a community population of diabetic patients. Diabetic Medicine, [S.L.], v. 15 , n. 2, p. 148-150, fev. 1998. Wiley. http:// dx.doi.org/10.1002/(sici)1096-

9136(199802)15:23.0.co;2-h. Acesso em: 06 out. 2021

SOUZA, Otton Luis Raffo et al. Prevalência de Autoimunidade Tireoidiana em Um Grupo de Pacientes Com Diabetes Mellitus Tipo 1 em Londrina, PR. 2004. Disponível em: https://www.scielo.br/scielo.php? pid=S0004-

27302005000200008\&script=sci abstract\&tlng =pt. Acesso em: 26 set. 2020.

VAN DEN DRIESSCHE A, Eenkhoorn V, Van Gaal L, De Block C. Type 1 diabetes and autoimmune polyglandular syndrome: a clinical review. Neth J Med. 2009 Dec; 67(11):376-87. PMID: 20009114. Disponível em: $\quad$ https://pubmed.ncbi.nlm.nih.gov/ 20009114/. Acesso em: 06 set 2021. 